\title{
Utilização de serviços de saúde por adolescentes rurais quilombolas e não quilombolas do semiárido baiano, Brasil
}

\author{
Health service utilization by Quilombola and non-Quilombola \\ adolescents living in a rural area in the semi-arid region of the state \\ of Bahia, Brazil
}

\author{
Katiuscy Carneiro Santana (https://orcid.org/0000-0001-6733-6631) ${ }^{1}$ \\ Etna Kaliane Pereira da Silva (https://orcid.org/0000-0002-2827-9395) ${ }^{2}$ \\ Raísa Brandão Rodriguez (https://orcid.org/0000-0003-0753-8906) ${ }^{3}$ \\ Vanessa Moraes Bezerra (https://orcid.org/0000-0001-5333-2875) ${ }^{3}$ \\ Raquel Souzas (https://orcid.org/0000-0002-7254-5486) ${ }^{3}$ \\ Danielle Souto de Medeiros (http://orcid.org/0000-0002-2480-8990) ${ }^{3}$
}

${ }^{1}$ Secretaria Municipal de Saúde, Prefeitura Municipal de Vitória da Conquista. Rua Coronel Gugé 211, Centro. 45040-150 Vitória da Conquista BA Brasil.

katiuscycs@gmail.com.

${ }^{2}$ Faculdade de Medicina,

Universidade Federal

de Minas Gerais. Belo

Horizonte MG Brasil.

${ }^{3}$ Instituto Multidisciplinar em Saúde, Universidade

Federal da Bahia. Salvador BA Brasil.

\begin{abstract}
This article describes health service utilization by Quilombola and non-Quilombola adolescents living in a rural area in the semi-arid region of Bahia. Quantitative and qualitative methods were used to gain a more in-depth understanding of the object of study. A cross-sectional household survey was conducted with 390 adolescents. Health service utilization was described using frequency distribution and 95\% confidence intervals. Discussions were held with four focus groups, which were transcribed and analyzed using content analysis. The most commonly reported usual place of care was the local family care center (70,0\%) and 15,1\% of the adolescents had sought health care in the last 15 days. The main reason for seeking care was illness (37,3\%). The findings of the qualitative component of the study reveal multiple factors influencing access to services, including long waiting times, lack of prioritization of adolescent care and geographical barriers. Health services should attempt to get closer adolescents and provide care tailored to the specific needs of this group.
\end{abstract}

Key words Adolescent, Rural health, Ethnicity and health, Access to health services
Resumo Este estudo descreve a utilização de serviços de saúde por adolescentes quilombolas e não quilombolas residentes em uma área rural do semiárido baiano. Métodos quantitativos e qualitativos foram utilizados para que se conseguisse uma maior aproximação com o objeto de análise. Foi realizado um estudo transversal com 390 adolescentes. A utilização de serviços de saúde foi descrita por meio da distribuição de frequências e respectivos intervalos de confiança 95\%. Formaram-se quatro grupos focais que foram transcritos $e$ analisados por meio da análise de conteúdo. A Unidade de Saúde da Família foi relatada como o serviço comumente procurado para atendimento de necessidades de saúde (70,0\%). A procura por serviços de saúde nos últimos 15 dias foi de 15,1\% e o principal motivo foi por doença (37,3\%). $O$ componente qualitativo revelou múltiplos contextos que influenciaram no acesso aos serviços, como elevado tempo de espera, falta de priorização nos atendimentos e barreiras geográficas. Os serviços de saúde devem estar mais próximos dos adolescentes com um cuidado em saúde que considere as particularidades desse grupo.

Palavras-chave Adolescente, Saúde da população rural, Origem étnica e saúde, Acesso aos serviços de saúde 


\section{Introdução}

A adolescência é definida como a fase da vida entre os 10 e 19 anos de idade e caracterizada como um período de transição entre a infância e a vida adulta ${ }^{1}$. A Constituição Brasileira ratifica o marco legal internacional na concepção de adolescentes como sujeitos de direitos, em condição peculiar de desenvolvimento, e o Estatuto da Criança e do Adolescente regulamenta e assegura o direito à saúde da criança e do adolescente, por intermédio do Sistema Único de Saúde (SUS)².

O Brasil é um país de dimensão continental e formação histórica e social diversificada, assim é necessário considerar que os adolescentes brasileiros se constituem grupo plural que não deve ser tratado numa perspectiva universal e homogênea. De modo que é importante reconhecer elementos relevantes na atenção à saúde do adolescente como classe social, gênero, raça, local de moradia, entre outros ${ }^{2,3}$.

Estudos mostram que o perfil de procura dos adolescentes por assistência à saúde possui características que os diferenciam do restante da população, como a não valorização de sintomas de menor gravidade e dificuldades em aproximar-se dos serviços de saúde com ações preventivas ${ }^{4,5}$. Distinções entre os gêneros são observadas, uma vez que as meninas procuram os serviços com demandas associadas à gravidez e parto $\mathrm{e}$, ainda $\mathrm{o}$ maior interesse e preocupação com a sua saúde $e^{6,7}$.

Os adolescentes residentes no meio rural convivem com dificuldades no acesso e limitações na qualidade dos serviços de saúde, assim como relativo isolamento geográfico ${ }^{8}$ e dinâmicas de vida que geram situações singulares que devem ser levadas em consideração na organização da atenção à saúde desses adolescentes ${ }^{3}$. Nesse contexto, consideremos as comunidades quilombolas que se distinguem por sua identidade étnica, com modos de vida singular, hábitos e aspectos sociais e históricos marcados por processos de opressão e resistência às violências estruturais, com uma alta prevalência de problemas básicos de saúde e dificuldades de acesso a bens e serviços ${ }^{1,9-12}$.

A informação sobre a atenção a saúde contextualizada no território é uma condição relevante e de vital importância para a sociedade e para o Estado no processo de gestão transformadora. Pois é no território que podemos compreender os processos e as tendências para diagnóstico das situações de risco social, além de possibilitar o monitoramento das ações executadas para redução das desigualdades e auxiliar na incorporação de estratégias intersetoriais ${ }^{13}$.
A utilização dos serviços de saúde pelos indivíduos representa o centro do funcionamento dos sistemas de saúde e é influenciada por características e necessidades dos indivíduos, assim como pela oferta dos serviços, facilidade de acesso, recursos financeiros, disponibilidade e vinculação dos profissionais de saúde ${ }^{14}$. Contudo, informações referentes à utilização dos serviços de saúde por adolescentes são menos encontradas na literatura, além disso, há certa negligência na busca de informações diversificadas no que tange a populações ditas minoritárias como os residentes em áreas rurais, principalmente os quilombolas ${ }^{3}$. Diante desse cenário, a realização deste estudo teve como objetivo descrever a utilização de serviços de saúde por adolescentes quilombolas e não quilombolas residentes em uma área rural do semiárido baiano.

\section{Métodos}

Este estudo integra a pesquisa "Adolescer: Saúde do Adolescente da Zona Rural e seus Condicionantes", realizada em 2015, com adolescentes residentes em comunidades rurais quilombolas reconhecidas pela Fundação Cultural Palmares ${ }^{15}$ e não quilombolas de Vitória da Conquista, BA. A pesquisa foi aprovada no Comitê de Ética em Pesquisa com seres humanos do Instituto Multidisciplinar em Saúde da Universidade Federal da Bahia e todos os participantes assinaram Termo de Consentimento Livre e Esclarecido ou Termo de Assentimento Livre e Esclarecido. A combinação de métodos quantitativos e qualitativos foi utilizada para que se conseguisse uma maior aproximação com o objeto de estudo.

\section{Componente quantitativo}

Trata-se de um estudo transversal, de base populacional e abordagem domiciliar realizado com adolescentes entre 10 e 19 anos de idade, residentes em 21 comunidades rurais (nove quilombolas), baseado na aplicação de questionário semiestruturado. $\mathrm{O}$ instrumento de coleta de dados foi elaborado a partir de inquéritos realizados no Brasil, através da Pesquisa Nacional de Saúde do Escolar (PeNSE) ${ }^{16}$ e Pesquisa Nacional de Saúde (PNS) ${ }^{17}$, que sofreu adaptações para adequá-lo ao contexto da zona rural e sua versão final foi elaborada após pré-testes e estudo piloto.

Para realizar a estimativa populacional, foram utilizados os dados da Ficha A preenchida pelos Agentes Comunitários de Saúde (ACS) durante 
as visitas domiciliares, confirmados em posterior mapeamento. $\mathrm{O}$ universo amostral foi composto por 811 adolescentes ( 350 quilombolas e 461 não quilombolas). No intuito de garantir a representatividade e a viabilidade da pesquisa, optou-se pelo cálculo de duas amostras, uma para os adolescentes quilombolas e outra para os adolescentes não quilombolas.

No cálculo amostral foi considerado: prevalência de $50 \%$, dada a heterogeneidade dos eventos mensurados; precisão de 5\%; nível confiança de 95\%; efeito de desenho igual a 1,0; e acréscimo de $15,0 \%$ para possíveis perdas. Entretanto, considerando que seria entrevistado apenas um adolescente por domicílio e que o número de domicílios para o estrato quilombola seria superado, foram acrescidos 7,1\% para perdas nesse estrato.

A amostragem para os não quilombolas deuse em duas etapas: 1) seleção aleatória de domicílios que continham adolescentes, de acordo com a distribuição proporcional de adolescentes por comunidade; 2) seleção aleatória dos adolescentes em cada domicílio. Foram entrevistados adolescentes em todos os domicílios quilombolas, procedendo-se apenas seleção aleatória dos adolescentes do domicilio.

O estudo piloto aconteceu em dezembro de 2014 em comunidade rural não participante do estudo principal. A pesquisa foi apresentada nas reuniões do Conselho Local de Saúde, Conselho Territorial Quilombola e das equipes da Estratégia de Saúde da Família (ESF), para apoio e divulgação nas comunidades. Por se tratar de uma região rural, foi realizado mapeamento dos domicílios e equipamento da comunidade. A sensibilização dos domicílios ocorreu simultaneamente ao mapeamento, com o auxílio dos ACS.

$\mathrm{O}$ questionário utilizado foi organizado em blocos temáticos que incluíram características sociodemográficas, comportamentos de risco e proteção para a saúde, entre os quais a utilização dos serviços de saúde foi abordada. A coleta de dados do campo principal ocorreu no período de janeiro a maio de 2015, por estudantes de graduação previamente treinados e utilização de computadores portáteis (HP Pocket Rx5710) como ferramenta de coleta. Para garantia da qualidade dos dados foram repetidas as entrevistas em 5\% da amostra.

Para descrever a utilização dos serviços de saúde dos adolescentes neste estudo, foram utilizadas as seguintes variáveis: procura mesmo lugar, médico ou serviço de saúde (não; sim); local que costuma buscar atendimento à saúde (farmácia; Unidade Básica de Saúde (UBS) / Unida- de de Saúde da Família (USF); pronto-socorro/ emergência de hospital público; hospital público/ambulatório; outros); última consulta médica (12 últimos meses; de um a menos de dois anos; dois anos ou mais / nunca foi ao médico); procura pelo serviço de saúde nos últimos 15 dias (não; sim); motivo de não procura ao serviço de saúde nos últimos 15 dias (não houve necessidade; o local de atendimento era distante ou de difícil acesso; horário incompatível; atendimento muito demorado; outro motivo); atendimento na primeira procura ao serviço de saúde (não, sim); local de procura do primeiro atendimento (UBS / USF; pronto-socorro/ emergência de hospital público; hospital público/ambulatório; outros); principal motivo de procura por serviços de saúde (acidente ou lesão; doença; problema odontológico; continuação de tratamento; vacinação; outros); prescrição de medicamento no último atendimento (não; sim); conseguiu os medicamentos receitados (todos; alguns; nenhum); como conseguiu os medicamentos (plano de saúde; programa farmácia popular; serviço público de saúde; mista; nenhuma acima); pagamento pelos medicamentos (não; sim); internação em hospital por 24 horas ou mais nos últimos 12 meses (não; sim); principal atendimento de saúde no internamento (parto normal; tratamento clínico; tratamento psiquiátrico; cirurgia; exames complementares de diagnóstico; outro); internação feita pelo SUS (não; sim); cobertura do plano de saúde no internamento (não; sim); pagamento por internação (não; sim).

Os dados foram descritos por meio de distribuição de frequências e respectivos intervalos de confiança 95\%. O programa Stata, versão 15.0, foi utilizado para as análises dos dados.

\section{Componente qualitativo}

Grupos focais com encontros realizados na tentativa de compreender a representação social dos adolescentes em relação às condições, acesso e utilização dos serviços de saúde. Foram realizados quatro grupos focais com meninos quilombolas e não quilombolas e meninas quilombolas e não quilombolas.

Os grupos foram criados em novembro de 2015, em uma escola municipal do distrito que reunia as comunidades rurais estudadas, sendo realizado um grupo por dia, com média de dez participantes e duração aproximada de $40 \mathrm{mi}$ nutos. O critério para inclusão no grupo foi este ter utilizado o serviço de saúde nos últimos doze meses; estudar na sede do distrito e estar com 
idade entre 12 e 18 anos. Depois dessa fase, seguiu-se ao sorteio aleatório entre os grupos. $\mathrm{Na}$ fase de sensibilização, cada adolescente sorteado foi visitado para esclarecimentos sobre o estudo e convidado a participar.

Após consentimento dos participantes, as conversas em grupo foram gravadas e digitalizadas. Para assegurar o anonimato desses adolescentes, os nomes utilizados são fictícios escolhidos por eles. Os tópicos em estudo foram abordados utilizando perguntas preestabelecidas em um roteiro semiestruturado.

Para análise dos dados, utilizou-se a técnica de Análise de Conteúdo proposta por Bardin ${ }^{18}$, que tem como característica analisar o que foi dito e observado nas entrevistas, classificando em temas ou categorias que auxiliem na compreensão do que está nos discursos. Para a condução da análise dos dados foram estruturadas três etapas, como assinala a técnica proposta: fase de pré-análise, descrição analítica e interpretação inferencial.

$\mathrm{Na}$ primeira fase, pré-análise, foi realizada a organização das informações coletadas, através da leitura "flutuante" das transcrições dos áudios para obter uma primeira impressão acerca das informações coletadas. Na segunda fase, exploração do material, os dados foram classificados e categorizados, considerando recortes dos transcritos e, na terceira fase, que compreende o tratamento dos resultados e interpretação, foi realizada uma abordagem por frequência dos dados para as devidas interpretações.

\section{Resultados}

\section{Componente quantitativo}

Foram entrevistados 390 adolescentes, dos quais $200(51,3 \%)$ eram meninas e $167(42,8 \%)$ eram quilombolas. As perdas foram de $15,2 \%$ e $7,9 \%$, nos estratos quilombola e não quilombola, respectivamente. Perdas diferenciais foram observadas com predominância de meninos não quilombolas (p-valor 0,038 ), os principais motivos para perdas foram: domicílio fechado após três visitas, adolescente não encontrado após três visitas e recusa.

A maioria dos adolescentes costumava procurar o mesmo lugar, médico ou serviço de saúde quando precisavam de atendimento de saúde (70,0\%), e a UBS/USF o serviço de saúde que costumavam procurar $(70,0 \%)$. Mais da metade dos entrevistados $(54,4 \%)$ consultaram um mé- dico nos 12 meses anteriores à entrevista. A procura por atendimento de saúde nos últimos 15 dias foi relatada por $15,5 \%$ dos adolescentes. Dos que não procuraram o serviço de saúde nos últimos 15 dias, a maioria não o fez por não haver necessidade $(92,2 \%)$ (Tabela 1$)$.

Dentre os adolescentes que procuraram algum atendimento de saúde, $96,6 \%$ conseguiram atendimento na primeira vez em que procuraram e $66,1 \%$ desses atendimentos foram realizados na UBS/USF. O principal motivo de procura por atendimento relatado foi doença $(37,3 \%)$. Entre aqueles que conseguiram atendimento de saúde, 59,7\% tiveram medicamentos prescritos e $79,4 \%$ conseguiram obter todos os medicamentos prescritos, sendo $12,5 \%$ e $15,6 \%$ através do serviço público de saúde e do Programa Farmácia Popular, respectivamente, 54,6\% dos adolescentes pagaram pelos medicamentos (parcial ou integralmente) (Tabela 2).

Dos entrevistados, $7,2 \%$ foram internados em hospitais por 24 horas ou mais nos últimos 12 meses. Tratamento clínico $(39,3 \%)$ e cirurgia $(14,3 \%)$ foram os dois tipos de atendimentos mais frequentes nos casos de internação. A maioria $(82,1 \%)$ dos adolescentes foram internados por meio do SUS (Tabela 3 ).

\section{Componente qualitativo}

Nos grupos focais, a maioria dos adolescentes fez uma referência positiva sobre a própria saúde e consideraram que, de modo geral, são saudáveis. Relataram que buscam a USF localizada na sede do distrito ou as unidades satélites da USF (postinhos) localizados nas comunidades, quando necessitam de atendimento à saúde, seja diretamente ou através do ACS. Consideraram que a USF é importante na medida em que ajudam as pessoas a resolverem seus problemas de saúde e necessária, principalmente, para aquelas com poucos recursos financeiros. Entretanto, houve distinção entre quilombolas e não quilombolas, em relação ao não acesso ao atendimento de saúde na USF. De modo que, os quilombolas buscaram preferencialmente os hospitais públicos e os não quilombolas as clínicas privadas.

O contato com o serviço de saúde deu-se, em sua maioria, por demanda espontânea, principalmente para vacinação, verificação de peso e altura, consulta médica e odontológica. Relataram também que tiveram acesso a algumas ações de saúde que a equipe da USF realizou na escola, a exemplo de atividades educativas, verificação e atualização do cartão vacinal, avaliação antro- 
Tabela 1. Descrição da utilização dos serviços de saúde pelos adolescentes quilombolas e não quilombolas residentes em uma área rural do semiárido baiano. Bahia, 2015. $(\mathrm{n}=390)$.

\begin{tabular}{|c|c|c|c|}
\hline Variáveis & n & $\%$ & IC95\% \\
\hline \multicolumn{4}{|l|}{$\begin{array}{l}\text { Procura o mesmo lugar, } \\
\text { médico ou serviço de saúde }\end{array}$} \\
\hline Não & 117 & 30,0 & $25,6-34,7$ \\
\hline Sim & 273 & 70,0 & $65.2-74.4$ \\
\hline \multicolumn{4}{|l|}{$\begin{array}{l}\text { Local que costuma buscar } \\
\text { atendimento à saúde }\end{array}$} \\
\hline Farmácia & 27 & 6,9 & $4,8-9,9$ \\
\hline $\begin{array}{l}\text { Unidade Básica de Saúde } \\
\text { / Unidade de Saúde da } \\
\text { Família }\end{array}$ & 273 & 70,0 & $65,4-74,5$ \\
\hline $\begin{array}{l}\text { Pronto-socorro / } \\
\text { emergência de hospital } \\
\text { público }\end{array}$ & 31 & 8,0 & $5,6-11,1$ \\
\hline $\begin{array}{l}\text { Hospital público / } \\
\text { ambulatório }\end{array}$ & 20 & 5,1 & $3,3-7,8$ \\
\hline Outros & 38 & 9,7 & $7,2-13,1$ \\
\hline \multicolumn{4}{|l|}{ Última consulta médica } \\
\hline 12 últimos meses & 212 & 54,4 & $49,5-59,4$ \\
\hline 1 ano a menos de 2 anos & 114 & 29,3 & $24,9-34,0$ \\
\hline $\begin{array}{l}2 \text { anos ou mais / nunca } \\
\text { foi ao médico }\end{array}$ & 63 & 16,2 & $12,8-20,2$ \\
\hline \multicolumn{4}{|l|}{$\begin{array}{l}\text { Procura pelo serviço de } \\
\text { saúde nos últimos } 15 \text { dias }\end{array}$} \\
\hline Não & 331 & 84,9 & $80,9-88,1$ \\
\hline Sim & 59 & 15,1 & $11,9-19,0$ \\
\hline \multicolumn{4}{|l|}{$\begin{array}{l}\text { Motivo de não procura ao } \\
\text { serviço de saúde nos últimos } \\
15 \text { dias }^{*}\end{array}$} \\
\hline Não houve necessidade & 305 & 92,2 & $88,7-94,6$ \\
\hline $\begin{array}{l}\text { O local de atendimento } \\
\text { era distante ou de difícil } \\
\text { acesso }\end{array}$ & 9 & 2,7 & $1,4-5,1$ \\
\hline Horário incompatível & 4 & 1,2 & $0,4-3,2$ \\
\hline $\begin{array}{l}\text { Atendimento muito } \\
\text { demorado }\end{array}$ & 5 & 1,5 & $0,6-3,6$ \\
\hline Outro motivo & 8 & 2,4 & $1,2-4,8$ \\
\hline
\end{tabular}

Valor de p calculado pelo qui-quadrado; Valor de p calculado pelo teste exato de Fischer; ${ }^{*} \mathrm{n}=331$.

Fonte: Elaborada pelos autores.

pométrica. As meninas apresentaram ainda, uma característica que difere dos meninos, como a preocupação com a saúde, evidenciada pelo relato de procura dos serviços para marcações de exames, consultas de prevenção/rotina e realização de exames para diagnóstico de problemas apresentados. Já os meninos relataram a necessidade de procurar o serviço por motivos de acidentes ou lesões.
Tabela 2. Características da utilização dos serviços de saúde nos últimos 15 dias pelos adolescentes quilombolas e não quilombolas residentes em uma área rural do semiárido baiano. Bahia, 2015 ( $\mathrm{n}=59)$.

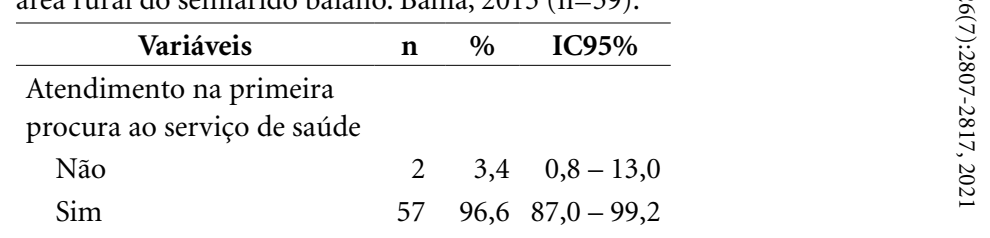

Local de procura do

primeiro atendimento

$\begin{array}{lccc}\begin{array}{l}\text { Unidade Básica de Saúde } \\ \text { / Unidade de Saúde da }\end{array} & 39 & 66,1 & 52,8-77,2 \\ \begin{array}{l}\text { Família } \\ \begin{array}{l}\text { Pronto-socorro ou } \\ \text { emergência de hospital } \\ \text { público }\end{array}\end{array} & 6 & 10,2 & 4,5-21,3 \\ \begin{array}{l}\text { Hospital público/ } \\ \text { ambulatório }\end{array} & 5 & 8,5 & 3,5-19,2 \\ \text { Outros } & 9 & 15,3 & 8,0-27,2\end{array}$

Principal motivo de procura por serviços de saúde

$\begin{array}{lrrr}\text { Acidente ou lesão } & 3 & 5,1 & 1,6-15,0 \\ \text { Doença } & 22 & 37,3 & 25,7-50,6 \\ \text { Problema odontológico } & 5 & 8,5 & 3,5-19,2 \\ \text { Continuação de } & 4 & 6,8 & 2,5-17,1 \\ \text { tratamento } & & & \\ \text { Vacinação } & 3 & 5,1 & 1,6-15,0 \\ \text { Outros } & 22 & 37,2 & 25,7-50,6\end{array}$

Prescrição de medicamento no último atendimento

$\begin{array}{llll}\text { Não } & 23 & 40,4 & 28,2-53,9 \\ \text { Sim } & 34 & 59,7 & 46,1-71,8\end{array}$

Conseguiu os

medicamentos receitados

Todos

Alguns

$27 \quad 79,4 \quad 61,6-90,2$

Nenhum

$6 \quad 17,7 \quad 7,8-35,2$

$1 \quad 2,9 \quad 0,4-19,8$

Como conseguiu os

medicamentos

$\begin{array}{lrrr}\text { Plano de saúde } & 3 & 9,3 & 2,9-26,6 \\ \text { Programa Farmácia } & 5 & 15,6 & 6,3-33,6 \\ \begin{array}{l}\text { Popular } \\ \text { Serviço público de saúde }\end{array} & 4 & 12,5 & 4,5-30,2 \\ \text { Mista } & 12 & 37,5 & 22,0-56,1 \\ \text { Nenhuma acima } & 8 & 25,0 & 12,5-43,7\end{array}$

Pagamento pelos

medicamentos

\begin{tabular}{llll} 
Não & 15 & 45,4 & $28,8-63,2$ \\
Sim & 18 & 54,6 & $36,8-71,2$ \\
\hline
\end{tabular}

Fonte: Elaborada pelos autores.

Com relação à ideia que os adolescentes têm sobre o serviço público de saúde, todos os grupos 
Tabela 3. Descrição de internação em hospital nos últimos 12 meses dos adolescentes quilombolas e não quilombolas residentes em uma área rural do semiárido baiano. Bahia, 2015 ( $\mathrm{n}=28)$.

\begin{tabular}{lrrr}
\hline \multicolumn{1}{c}{ Variáveis } & n & \% & \multicolumn{1}{c}{ IC95\% } \\
\hline Internação em hospital & & & \\
por 24 horas ou mais nos & & & \\
últimos 12 meses (n=390) & & & \\
$\quad$ Não & 362 & 92,8 & $89,8-95,0$ \\
$\quad$ Sim & 28 & 7,2 & $5,0-10,2$ \\
Principal atendimento de & & & \\
saúde no internamento & & & \\
$\quad$ Parto normal & 2 & 7,1 & $1,6-26,2$ \\
$\quad$ Tratamento clínico & 11 & 39,3 & $22,4-59,2$ \\
$\quad$ Tratamento psiquiátrico & 2 & 7,1 & $1,6-26,2$ \\
$\quad$ Cirurgia & 4 & 14,3 & $5,1-33,9$ \\
$\quad$ Exames complementares & 2 & 7,1 & $16,3-26,2$ \\
$\quad$ de diagnóstico & & & \\
$\quad$ Outro & 7 & 25,0 & $11,8-45,3$ \\
Internação feita pelo SUS & & & \\
$\quad$ Não & 5 & 17,9 & $7,2-37,9$ \\
$\quad$ Sim & 23 & 82,1 & $62,1-92,8$ \\
Cobertura do plano de & & & \\
saúde no internamento & & & \\
$\quad$ Não & 26 & 92,9 & $73,7-98,4$ \\
$\quad$ Sim & 2 & 7,1 & $1,6-26,3$ \\
Pagamento por internação & & & \\
$\quad$ Não & 24 & 85,7 & $66,0-94,9$ \\
$\quad$ Sim & 4 & 14,3 & $5,1-34,0$ \\
\hline Fonte: Elaborada pelos autores. & & &
\end{tabular}

relataram que a demora, tanto para realização de consultas como marcações de exames, principalmente na atenção especializada, muitas vezes é associada à "falta de prioridade" para atendimentos dos adolescentes: "Só atende mulher grávida", "povo mais velho" e "crianças para vacinar, pesar", mostrando dificuldade para acesso aos serviços de saúde. Fizeram referência também sobre a estrutura física, conservação dos equipamentos e higienização da USF e unidades satélites, relatando que possuem estrutura precária, com equipamentos mal conservados e com um ambiente que mereceria maior rigor de higienização.

Um aspecto levantado, apenas pelos não quilombolas, foi a distância do local de residência ao centro urbano, aliado à pouca oferta de transporte noturno e ao horário restrito de funcionamento da USF, fatores estes que dificultavam o acesso, principalmente aos cuidados de urgência e emergência em saúde. Entre os quilombolas foi pontuada a frequência com que ocorrem os atendimentos em saúde nas comunidades quilombolas: eles relataram que os atendimentos acontecem normalmente uma vez por mês, o que restringe ainda mais o acesso da população aos cuidados em saúde.

\section{Discussão}

Os adolescentes rurais estudados costumavam procurar a USF quando necessitavam de cuidados em saúde. Os serviços de saúde foram utilizados, em sua maioria, por meio do SUS. Dificuldades foram mencionadas em relação ao elevado tempo de espera por atendimento, à falta de prioridade para os adolescentes, precária estrutura física e de conservação das unidades de saúde e baixa regularidade de oferta de atendimento nas comunidades. Quando não foram atendidos na USF, os adolescentes quilombolas e não quilombolas relataram diferentes estratégias na busca por cuidados em saúde. Os quilombolas procuravam preferencialmente os hospitais públicos $\mathrm{e}$ os não quilombolas as clínicas privadas. Houve baixa procura por serviços de saúde nos 15 dias anteriores à entrevista.

Quase a totalidade $(96,6 \%)$ dos adolescentes que necessitaram de atendimento, conseguiram obter na primeira busca, semelhante ao encontrado na população brasileira $(95,3 \%)^{19}$. Essas experiências podem interferir diretamente nas próximas decisões de procura pelo mesmo lugar, médico ou serviço de saúde, articulando-se com a ideia de que consultar um profissional ou serviço de saúde definido relaciona-se a um melhor acesso aos serviços, sendo considerado como um elemento importante da qualidade da atenção, continuidade da assistência e relação entre o profissional de saúde e o adolescente ${ }^{4}$

Nesse sentido, a Estratégia de Saúde da Família (ESF) necessita de diretrizes que apoiem diferentes atividades a elas relacionadas, fortalecendo o acolhimento e o vínculo com a comunidade, trazendo ao adolescente segurança e referência de atendimento em saúde, potencializando mudanças positivas com relação ao cuidado em saúde, visto que eles demonstram conhecimento sobre os serviços ofertados na comunidade e a dinâmica dos lugares e dos sujeitos (individual e coletivo), o que garante a continuidade e a resolutividade das ações de saúde e a longitudinalidade do cuidado.

Pra cuidar da saúde das pessoas. Cuidar da saúde das pessoas necessitadas. Pra marcar algum exame. (Bagunça, não quilombola). 
Para a doação de alguns remédios. (Marrentinha, não quilombola).

Pra ajudar as pessoas, com seus problemas. (Estrela, quilombola).

O SUS visa assegurar atenção universal, equitativa e integral aos indivíduos, sendo observado no estudo que a USF e unidades satélites foram os serviços mais procurados pela grande maioria $(70,0 \%)$ dos adolescentes, compatíveis com outros levantamentos de saúde da população brasileira $^{19,20}$, com estimativas de $56,9 \%$ e $47,9 \%$ respectivamente e ainda de outras comunidades rurais (Jequitinhonha, MG) $87,4 \%{ }^{21}$.

Na maioria das coisas, sempre quando ocorre uma doença é direto pro posto. (Narizinho, quilombola).

(O que você faz quando precisa de atendimento?) Procura o médico (...) no posto. (Caxixe, quilombola).

(O que você faz quando precisa de atendimento?) Pede pra agente de saúde marcar um exame (no posto) (Patrick, não quilombola).

A maior procura por Unidades de Saúde, por meio da ESF, é reforçada pelo grande peso destes tipos de serviços como porta de entrada, especialmente no setor público nas zonas rurais, onde outros tipos de acesso ao serviço são mais escassos em relação ao âmbito urbano ${ }^{21}$. Além disso, ter um serviço de escolha para os adolescentes pode estar relacionado à atenção, experiências positivas de atendimento e vínculo de confiança com os profissionais, muitas vezes, por já conhecê-los na comunidade ${ }^{22}$.

Apesar de estudos apontarem avanços no acesso e utilização dos serviços de saúde no Brasil, principalmente relacionados à ampliação da oferta de serviços na rede básica de saúde, ainda se observam, em grupos minoritários, desigualdades geográficas e sociais ${ }^{6,23}$. Porém, há de se considerar que uma parcela $(7,8 \%)$ dos adolescentes, apesar de sentir necessidade de atendimento à saúde, não o procura.

O hábito de procurar ou não serviços de saúde em casos de necessidade pode estar relacionado tanto aos fatores culturais quanto às dificuldades para o acesso aos serviços. Os adolescentes relataram que o principal motivo é por não haver necessidade; mas também, afirmaram não procurar o serviço devido à distância geográfica característico das zonas rurais, horários incompatíveis e atendimento demorado.

... já teve caso de uma amiga minha voltar pra casa e não ser atendida... tinha muita gente, $e$ olha ela foi uma das primeiras a chegar, e demorou muito pra atender ela (Rosa - quilombola).
Às vezes é bom, mais... demora muito às vezes, quando é exame (Capacete - não quilombola).

É bom, só que é muito ruim porque demora demais, porque tipo, quando a gente vai pedir pra fazer os exames, demora muito pra chegar. Então é melhor ir a Vitória da Conquista porque vinha aqui e demorava. Uma vizinha da nossa fazenda demorou seis meses, aí quando foi ver ela já tinha morrido, aí depois que foi chegar os exames (Adolescente não identificada, quilombola)

Uma barreira que parece dificultar o acesso e utilização dos serviços de saúde pelos adolescentes é o tempo de espera elevado nos atendimentos, mostrando também que o planejamento estruturado e típico das unidades não abrange todos os públicos, ou seja, não são considerados grupo prioritário nos atendimentos ofertados pela ESF, situação que também foi evidenciada em outro estudo com adolescentes baianos ${ }^{24}$.

...só atende mulher grávida...e o povo mais velho. (Majin Boo, quilombola).

Crianças pra vacinar, pesar. (Kel, quilombola).

E o povo doente, que anda com muleta. (Caxixe, quilombola).

Às vezes tem esse problema, a gente precisa ir pra escola, ai o dia é marcado pra gente de manhã, ai a gente vai, né. Quando chegamos lá, chegamos até sendo primeiro só que tem que colocar criança na frente, gestantes, idosos, ai a gente fica por último, ai às vezes não somos atendidos, por que temos que voltar pra casa, pra ir pra escola, ai fica sem atendimento. (Estrela, quilombola).

Os adolescentes ainda evidenciaram a precariedade de recursos do serviço de saúde público, e fizeram referência sobre a estrutura física precária e a conservação dos equipamentos da USF e unidades satélites.

Tem que vir aqui, tem posto lá que só abre de mês em mês, tá tudo sujo. (Caxixe, quilombola).

$A h$, alguns postos, ferrugem que comem os armários, essas coisas(...). (Batoré, não quilombola).

Os adolescentes quilombolas e não quilombolas relataram comportamentos diferentes quando não foram atendidos na USF: os quilombolas, preferencialmente, buscavam por cuidados em saúde nos hospitais públicos e os não quilombolas, em sua maioria, nas clínicas privadas.

Aí era pra ser pelo SUS, só que demorou, aí teve que pagar. (Bin Laden, não quilombola).

Assim eu senti muita pontada no peito, ai ela foi aumentando, aumentando, efui internada duas vezes [...]. foi, foi em Conquista! Serviço público. (Princesinha, quilombola).

Vou, geralmente, no particular. (Adolescente não identificada, não quilombola). 
Eu também fui passar na ginecologista. Quando eu passei lá (no particular) demorou seis meses aí que foi chegar o daqui (do posto). (Menorzinha, não quilombola).

Depende das condições da pessoa, né? Às vezes vai pelo SUS ou vai particular. (Bolachão, não quilombola).

Estudos com adolescentes evidenciam que fatores socioeconômicos implicam no acesso aos serviços de saúde ${ }^{4,25}$, e entre os quilombolas a parcela de adolescente em níveis econômicos mais baixos foi maior do que entre os não quilombolas $(76,1 \% \text { e } 50,2 \% \text {, respectivamente })^{26}$. Claro et al..$^{25}$ evidenciam que os estudantes de escola pública têm quase cinco vezes mais chance de buscar uma unidade básica de saúde do que os adolescentes de escola privada, podendo-se considerar maior facilidade de acesso destes últimos aos planos privados e/ou serviços particulares. Nunes et al. ${ }^{4}$ abordam iniquidade em saúde, ao identifica menor prevalência de uso dos serviços de saúde por adolescentes de classes socioeconômicas mais baixas, que trabalhavam e que não estudavam e que referiram cor da pele preta.

Outras dificuldades de acesso pontuadas, mas apenas pelos adolescentes não quilombolas, foram a distância do local de residência ao centro urbano, pouca oferta de transporte noturno e horário de funcionamento da USF. Fatores que dificultam o acesso principalmente nas situações urgência e emergência.

É porque lá (zona urbana) tudo é mais perto, aqui tudo é mais longe. (Bin Laden, não quilombola).

Então, tipo assim, como de madrugada aqui não tem atendimento, a não ser que vá pro hospital [...] e não achava transporte porque o ônibus não "tava" rodando, era duas e pouca da manhã aí ou ia de pé ou então morria. (Estrela, não quilombola).

Todavia, os adolescentes quilombolas não fizeram referência quanto à distância ou a pouca oferta de transporte para conseguir cuidados em saúde. Pode-se sugerir que esses aspectos não são relevantes para esse grupo ou que possuem maior capacidade de resiliência, adaptando-se às situações adversas, ou por estarem resignados diante das dificuldades vivenciadas rotineiramente.

A equidade no acesso e na utilização dos serviços de saúde implica na maneira como o serviço se organiza para minimizar as barreiras de acesso da população ${ }^{27}$. Nesse sentido, a equipe da USF quando se desloca até a localidade onde residem as pessoas, está tentando reduzir a barreira geográfica de acesso, com vistas a facilitar o uso dos serviços de saúde. Por outro lado, quando oferta um atendimento com a mesma frequência para localidades mais próximas ou mais longínquas, com perfis raciais e socioeconômicos diferentes, pode produzir iniquidades no acesso e na utilização dos serviços de saúde. As falas destacadas abaixo por adolescentes quilombolas sobre as barreiras de atendimento em suas comunidades reforça essa reflexão:

Só atende de vez em quando, não é direto não. (Madinbu, quilombola).

(Atendimento) de mês em mês. (Caxixe, quilombola).

Quando a gente tem um problema assim como você falou, a gente tem que vir, porque lá no posto só algumas vezes, de mês em mês. (Estrela, quilombola).

A esse respeito, Travassos e Castro ${ }^{27}$ enfatizam que situações de injustiça social são caracterizadas por desigualdades sociais nas condições de saúde e no acesso e na utilização de serviços, resultante de oportunidades diferenciadas em função da posição social dos indivíduos. Ademais, é relevante pontuar que tanto as diretrizes nacionais para atenção integral a saúde dos adolescentes ${ }^{1}$, quanto à política nacional de saúde integral da população negra ${ }^{9}$, destacam a necessidade de ações estratégicas para garantir e ampliar a atenção à saúde da população quilombola no intuito de reduzir as desigualdades étnico-raciais.

Observamos baixa prevalência de utilização dos serviços de saúde por questões preventivas, resultado corroborado pela literatura, a qual evidencia que os aspectos relacionados à doença são os motivos mais frequente de demanda aos serviços de saúde ${ }^{20,28}$.

Só vou quando eu "tô" doente. (Lua, quilombola).

Diniz afirma que a resistência do adolescente em aproximar-se dos serviços de saúde por ações preventivas pode associar-se com fatores como a não valorização dos sintomas que não sejam muito graves e que não sejam físicos.

"Tava" com dor no dente, aî eu fui. (Obama, não quilombola).

"Tava" com dor na barriga, dor na perna, dor nas costas... procurei o médico. (Safadão, não quilombola).

No entanto, alguns discursos mostraram que o serviço também foi utilizado com a finalidade de prevenção de agravos, principalmente quando o adolescente tinha parente ou familiar com patologias estabelecidas.

Eu só fiz um check-up aí um dia. (...) É porque meu pai têm (problema de saúde) e eu podia ter, entendeu!?". (Bin Laden, não quilombola). 
Ainda, mais da metade dos atendimentos realizados $(59,7 \%)$ tiveram prescrições de medicamentos. Para a população brasileira em geral, esse achado equivale a $64,8 \%{ }^{19}$. Este resultado pode estar relacionado à crescente "cultura" da medicalização, em que a maioria das consultas é finalizada com uma prescrição, visto que a população valoriza e prefere as práticas curativas ${ }^{29}$.

A prevalência do acesso a todos os medicamentos prescritos $(79,4 \%)$ foi consistente com a média nacional $(75,8 \%)^{19}$. Ressalta-se que apesar do programa da farmácia popular ter sido o meio mais frequente para obtenção dos medicamentos $(15,6 \%)$ seguido do serviço público de saúde $(12,5 \%)$, uma parcela importante $(54,6 \%)$ dos adolescentes pagou por esses medicamentos. Cabe dizer que a população não dispõe de farmácias pelo setor público nas regiões rurais, senão nas USF, logo a falta do medicamento incorre em impossibilidade da terapêutica indicada ou resulta em despesas para o custeio tanto para a compra dos medicamentos quanto para o deslocamento necessário para efetuá-la. Tal deficiência pode ocasionar agravamento de sintomas de saúde, determinando outras necessidades de procura ou diminuição das possibilidades de futuras buscas por serviços de saúde em virtude da experiência negativa vivenciada ou, ainda, a adoção de outras práticas curativas ${ }^{25}$.

Diante das dificuldades de acesso aos serviços de saúde que podem ser encontradas na região rural, $7,2 \%$ dos adolescentes precisaram de internação hospitalar nos 12 meses anteriores à entrevista. Vieira ${ }^{21}$ sugeriu que a alta prevalência de internações hospitalares pode estar relacionada a problemas de acesso à Atenção Básica, ou até mesmo ser reflexo da não adesão aos tratamentos relacionados à saúde. A maioria das internações foram para tratamento clínico $(39,3 \%)$ que, em geral, são decorrentes de complicações e agravamento de doenças crônicas, reforçando a necessidade de um melhor acesso aos cuidados primários para assim evitar a necessidade de cuidados agudos.

O presente estudo apresenta limitações, o tamanho da amostra do componente quantitativo não foi planejado para testar diferenças segundo gênero e local de residência, assim não foi possível estimar diferenças quantitativas entre os grupos.

\section{Considerações finais}

Por saber que os adolescentes passam por uma fase em que se constroem hábitos relacionados à saúde, os serviços devem estar cada vez mais próximos desse grupo, assumindo ações de educação e promoção de saúde centradas nas necessidades percebidas pelos adolescentes em seus diferentes contextos culturais e particularidades. Nesta perspectiva, diversas estratégias podem ser adotadas, por exemplo: ações intersetoriais tendo por base o território local; incorporação e ampliação de educação aos pares; e alteração na imagem social dos serviços de saúde que por vezes são vistos como não acolhedores aos adolescentes ${ }^{30}$.

A integralidade da atenção nos serviços de Atenção Básica à Saúde pode gerar um impacto importante na saúde dos adolescentes brasileiros, em especial dos adolescentes rurais e/ou quilombolas. De modo que, o fortalecimento da atenção básica e, principalmente, da Estratégia Saúde da Família torna-se fundamental para um melhor desempenho dos serviços voltados à saúde dos adolescentes.

O acesso aos serviços de saúde é um direito assegurado pelo SUS, portanto, quando se obstruiu o acesso está também violando esse direito, comprometendo a assistência adequada em tempo oportuno. Os resultados desse artigo são relevantes para o fornecimento de informações que contribuam para a definição de estratégias efetivas de atenção à saúde do adolescente. 


\section{Colaboradores}

KC Santana, EKP Silva, RB Rodriguez, R Souzas e DS Medeiros participaram da concepção do estudo, implementação do mesmo, análise e interpretação dos resultados e redação do artigo. VM Bezerra participou da concepção do estudo, interpretação dos resultados e realizou análise crítica relevante do conteúdo intelectual. Todas as autoras leram e aprovaram a versão final desse manuscrito.

\section{Agradecimentos}

Às famílias e adolescentes rurais, entrevistadores, Agentes Comunitários de Saúde e demais profissionais de saúde e da educação fundamentais na execução desse trabalho. À Fundação de Amparo à Pesquisa do Estado da Bahia (FAPESB) e à Coordenação de Aperfeiçoamento de Pessoal de Nível Superior (CAPES) pelo financiamento.

\section{Referências}

1. Brasil. Ministério da Saúde (MS). Secretaria de Atenção à Saúde. Departamento de Ações Programáticas Estratégicas. Diretrizes nacionais para a atenção integral à saúde de adolescentes e jovens na promoção, proteção e recuperação da saúde. Brasília: MS; 2010.

2. Brasil. Constituição (2012). Lei no 8.069, de 13 de Julho de 1990, e Legislação Correlata no 8069, de 2012. Estatuto da Criança e do Adolescente. $9^{\mathrm{a}}$ ed. 2012

3. Brasil. Ministério da Saúde (MS). Secretaria de Atenção à Saúde. Departamento de Ações Programáticas e Estratégicas. Proteger e cuidar da saúde de adolescentes na atenção básica. Brasília: MS; 2017.

4. Nunes BP, Flores TR, Duro SMS, Saes MO, Tomasi E, Santiago AD, Thumé E, Facchini LA. Utilização dos serviços de saúde por adolescentes: estudo transversal de base populacional, Pelotas-RS, 2012. Epidemiol Serv Saude 2015; 24(3):411-420.

5. Diniz LR, Oliveira PF. Discursos de Adolescentes da zona rural sobre a Adolescência [dissertação]. Pernambuco: Universidade Federal de Pernambuco; 2010.

6. Pinheiro RS, Viacava F, Travassos C, Brito AS. Gênero, morbidade, acesso e utilização de serviços de saúde no Brasil. Cien Saude Colet 2002; 7(4):687-707.

7. Levorato CD, Mello LM, Silva AS, Nunes AA. Fatores associados à procura por serviços de saúde numa perspectiva relacional de gênero. Cien Saude Colet 2014; 19(4):1263-1274.

8. Brasil. Ministério da Saúde (MS). Secretaria de Gestão Estratégica e Participativa. Departamento de Apoio à Gestão Participativa. Política Nacional de Saúde Integral das Populações do Campo e da Floresta. Brasília: MS; 2013.

9. Brasil. Ministério da Saúde (MS). Portaria MS/GM no 992, de 13 de maio de 2009. Institui a Política Nacional de Saúde Integral da População Negra. Diário Oficial da União 2009; 14 maio.

10. Freitas DA, Caballero AD, Marques AS, Hernández CIV, Antunes SLN. Saúde e comunidades quilombolas: uma revisão da literatura. Rev CEFAC 2011; 13(5):937-943

11. Bezerra VM, Medeiros DS, Gomes KO, Souzas R, Giatti L, Steffens AP et al. Inquérito de Saúde em Comunidades Quilombolas De Vitória da Conquista, Bahia, Brasil (Projeto COMQUISTA): Aspectos metodológicos e análise descritiva. Cien Saude Colet 2014; 19(6):1835-1847.

12. Silva EKP, Medeiros DS, Martins PC, Sousa LA,Lima GP, Rêgo MAS , Silva TO, Freire AS, Silva FM. Insegurança alimentar em comunidades rurais no Nordeste brasileiro: faz diferença ser quilombola?. Cad Saude Publica 2017; 33(4):e00005716.

13. Rigotto RM, Augusto LGS. Saúde e ambiente no Brasil: desenvolvimento, território e iniqüidade social. Cad Saude Publica 2007; 23(4):S475-S485.

14. Travassos C, Martins M. Uma revisão sobre os conceitos de acesso e utilização de serviços de saúde. Cad Saude Publica 2004; 20(Supl. 2):S190-S198. 
15. Fundação Cultural Palmares. Certidões expedidas às comunidades remanescentes de quilombos (CRQs) atualizada até a portaria no 268/2017. 2017. [acessado 2017 out 22]. Disponível em: http://www.palmares. gov.br/

16. Instituto Brasileiro de Geografia e Estatística (IBGE). Questionário PENSE 2012 [internet]. Rio de Janeiro: IBGE; 2012[acessado 2014 Jun 16]. Disponível em: http://biblioteca.ibge.gov.br/biblioteca-catalogo?view $=$ detalhes\&id $=52908$.

17. Pesquisa Nacional de Saúde (PNS). Questionário do Domicílio [Internet]. 2013 [acessado 2014 Jun 16]. Disponível em: http://www.pns.icict.fiocruz.br/arquivos/ Domiciliar/Modulo\%20A-PNS.pdf

18. Bardin L. Análise de conteúdo. $4^{\mathrm{a}}$ ed. Lisboa: Edições 70;2009.

19. Instituto Brasileiro de Geografia e Estatística (IBGE). Pesquisa nacional de saúde 2013 - Acesso e utilização dos serviços de saúde, acidentes e violências: Brasil, grandes regiões e unidades da federação. Rio de Janeiro: IBGE; 2015.

20. Instituto Brasileiro de Geografia e Estatística (IBGE). Pesquisa nacional de saúde do escolar: 2015. Rio de Janeiro: IBGE; 2016.

21. Vieira EWR. Acesso e utilização dos serviços de saúde de atenção primária em população rural do município de Jequitinhonha, Minas Gerais [dissertação]. Belo Horizonte: Universidade Federal de Minas Gerais; 2010.

22. Alves MCGP, Alves OSF, Bousquat A, Escuder MML, Segri NJ. Acesso aos serviços de saúde em Municípios da Baixada Santista. In: Uso de serviços de saúde. São Paulo: Instituto de Saúde; 2008. p.65-84.

23. Gomes KO, Reis EA, Guimarães MDC, Cherchiglia ML. Utilização de serviços de saúde por população quilombola do Sudoeste da Bahia, Brasil. Cad Saude Publica 2013; 29(9):1829-1842.

24. Martins MMF, Aquino R, Pamponet ML, Pinto Junior EP, Amorim LDA. Acesso aos serviços de atenção primária à saúde por adolescentes e jovens em um município do Estado da Bahia, Brasil. Cad Saude Publica 2019; 35(1):e00044718.

25. Claro LBL, March C, Mascarenhas MTM, Castro IAB, Rosa MLG. Adolescentes e suas relações com serviços de saúde: estudo transversal em escolares de Niterói, Rio de Janeiro, Brasil. Cad Saude Publica 2006; 22(8):1565-1574.

26. Sousa BC, Santos RS, Santana KC, Souzas R, Leite ÁJM, Medeiros DS. Comportamento sexual e fatores associados em adolescentes da zona rural. Rev Saude Publica 2018; 52:39.

27. Travassos C, Castro MSM. Determinantes e Desigualdades Sociais no Acesso e na Utilização de Serviços de Saúde. In: Giovanella L, Sarah E, Lobato LVC, Noronha JC, Carvalho AI, organizadores. Políticas e Sistemas de Saúde no Brasil. Rio de Janeiro: Editora Fiocruz; 2008.
28. Palazzo LS, Béria JU, Tomasi E. Adolescentes que utilizan servicios de atención primaria: ¿Cómo viven? ¿Por qué buscan ayuda y cómo se expresan? Cad Saude Publica 2003; 19(6):1655-1665.

29. Arrais PSD, Brito LL, Barreto ML, Coelho HLL. Prevalência e fatores determinantes do consumo de medicamentos no Município de Fortaleza, Ceará, Brasil. Cad Saude Publica 2005; 21(6):1737-1746.

30. Calazans G. Por Horizontes Mais Saudáveis. Revista Onda Jovem [periódico de Internet]. 2006 mar-jun [acessado 2021 abr 4]; 2(4):34-37. Disponível em: https: //issuu.com/ondajovem.com.br/docs/oj4.
Artigo apresentado em 30/05/2020

Aprovado em 29/04/2021

Versão final apresentada em 01/05/2021

Editores-chefes: Romeu Gomes, Antônio Augusto Moura da Silva 
